\title{
Mogamulizumab for Previously Treated Mycosis Fungoides and Sézary Syndrome: An Evidence Review Group Perspective of a NICE Single Technology Appraisal
}

\author{
Sabine E. Grimm ${ }^{1} @ \cdot$ Willem Witlox $^{1} \cdot$ Robert Wolff $^{2} \cdot$ Annette Chalker $^{2} \cdot$ Mickael Hiligsmann $^{3} \cdot$ Ben Wijnen $^{1}$. \\ Charlotte Ahmadu ${ }^{2} \cdot$ Steve Ryder $^{2} \cdot$ Nigel Armstrong $^{2} \cdot$ Steven Duffy ${ }^{2} \cdot$ Isabel Syndikus $^{4} \cdot$ Jos Kleijnen $^{2,3}$. \\ Manuela A. Joore ${ }^{1}$
}

Accepted: 26 September 2021 / Published online: 19 October 2021

(c) The Author(s) 2021, Corrected Publication 2022

\begin{abstract}
The National Institute for Health and Care Excellence (NICE) invited the manufacturer (Kyowa Kirin) of mogamulizumab $\left(\right.$ Poteligeo ${ }^{\circledR}$ ), as part of the single technology appraisal process, to submit evidence for its clinical and cost-effectiveness for previously treated mycosis fungoides (MF) and Sézary syndrome (SS). Kleijnen Systematic Reviews Ltd, in collaboration with Maastricht University Medical Centre, was commissioned to act as the independent evidence review group (ERG). This paper summarises the company submission (CS), presents the ERG's critical review of the clinical and cost-effectiveness evidence in the CS, highlights the key methodological considerations and describes the development of the NICE guidance by the Appraisal Committee. Based on a systematic literature review, one randomised controlled trial, MAVORIC, was identified showing favourable results in patients with MF and SS. However, MAVORIC compared mogamulizumab to vorinostat, which is not standard care in the NHS, and there is uncertainty due to the study design, specifically crossover of patients. Based on a "naïve comparison of results from the vorinostat arm of the MAVORIC study and the physician's choice arm (methotrexate or bexarotene i.e. United Kingdom [UK] standard treatments) of the ALCANZA study as well as comparison to Phase II bexarotene data", the company considered vorinostat to be "a reasonable proxy for current standard of care in the NHS". The ERG considered, based on the limited data available, that the comparability of vorinostat (MAVORIC) and physician's choice (ALCANZA) could not be established. In response to the Appraisal Consultation Document, the company provided an unanchored matched adjusted indirect comparison (MAIC) of mogamulizumab with UK standard care by analysing Hospital Episode Statistics (HES) data. However, given the high risk of bias of an unanchored MAIC, these results needed to be regarded with a considerable degree of caution. The economic analysis suffered from uncertainty because there was no trial evidence on the comparator in the England and Wales National Health Service (NHS), and it was unclear to what extent the trial (MAVORIC) comparator (vorinostat) was comparable to standard care, referred to as established clinical management (ECM) in the NHS. The evidence for overall survival had not reached maturity and was confounded by treatment switching, for which different crossover adjustment methods produced large variations in life years. Caregiver utilities were applied in the analysis, but there was a lack of guidance on their application and whether these were indicated in this appraisal. After consultation, the company updated the economic analysis with the MAIC. Incremental cost-effectiveness ratios comparing mogamulizumab against ECM were (depending on whether the HES or MAVORIC comparison were used) $£ 31,030$ or $£ 32,634$ per quality-adjusted life years (QALYs) gained according to the company's base case and $£ 38,274$ or $£ 80,555$ per QALY gained according to the ERG's base case. NICE did not recommend mogamulizumab for treating MF or SS in adults who have had at least one previous systemic treatment. This decision was subsequently appealed, and an appeal decision has been reached.
\end{abstract}

Extended author information available on the last page of the article 


\section{Key Points for Decision Makers}

National Institute for Health and Care Excellence (NICE) did not recommend mogamulizumab for treating mycosis fungoides or Sézary syndrome in adults who have had at least one previous systemic treatment.

Guidance is needed on when and how caregiver health effects should be included in economic evaluations.

A relevant comparator establishing the relative treatment effect should be included, for example, through indirect comparisons with available observational data, but potential biases inherent to these indirect comparisons need to be considered.

Crossover adjustment methods can lead to extreme difference in survival estimates. Full documentation on analyses performed is essential, and further guidance on the choice of the most suitable crossover adjustment method may be helpful.

\section{Introduction}

Mogamulizumab, trade name Poteligeo ${ }^{\circledR}$, was appraised within the National Institute for Health and Care Excellence (NICE) single technology appraisal (STA) process. Health technologies must be shown to be clinically effective and to represent a cost-effective use of National Health Service (NHS) resources in order to be recommended by NICE. Within the STA process, the company (Kyowa Kirin) provided NICE with a written submission and a mathematical health economic model, summarising the company's estimates of the clinical effectiveness and costeffectiveness of mogamulizumab for previously treated mycosis fungoides (MF) and Sézary syndrome (SS), an aggressive form of cutaneous T-cell lymphoma (CTCL) characterised by the presence of malignant lymphocytes called "Sézary cells" in the peripheral blood. This company submission (CS) was reviewed by an evidence review group (ERG) independent of NICE. The ERG, Kleijnen Systematic Reviews in collaboration with Maastricht University Medical Centre, produced an ERG report [1]. After consideration of the evidence submitted by the company and the ERG report, the NICE Appraisal Committee (AC) issued guidance whether or not to recommend the technology by means of the Final Appraisal Determination (FAD), which is open for appeal. This paper presents a summary of the ERG report and the development of the NICE guidance. Furthermore, it highlights important methodological issues that were identified, which may help in future decision making. Appeals were filed by several organisations, and the appeal decision has been reached.

Full details of all relevant appraisal documents (including the appraisal scope, CS, ERG report, consultee submissions, Appraisal Consultation Document (ACD), FAD, and comments from consultees) can be found on the NICE website [1].

\section{The Decision Problem}

The CS defined the population as "adults with advanced MF or SS CTCL (i.e. stage $\geq$ IIB MF and all SS) following at least one prior systemic therapy who are clinically ineligible for or refractory to treatment with brentuximab vedotin (BV)". This population was narrower than the population defined in the scope issued by NICE ("Adults with mycosis fungoides or Sézary syndrome cutaneous T-cell lymphoma following at least one prior systemic therapy") as it only included (1) adults with advanced MF or SS and (2) patients "who are clinically ineligible for or refractory to treatment with BV".

The intervention defined in the CS is in line with the NICE scope, i.e. mogamulizumab. Similarly, the CS covered the outcomes defined by NICE (i.e. progression-free survival [PFS], response rates, overall survival [OS], time to next treatment [TTNT], health-related quality of life [HRQoL], adverse effects of treatments).

The CS defined the comparator to be "established clinical management without mogamulizumab" (ECM), which is in line with the NICE scope. However, MAVORIC, the only randomised controlled trial (RCT) evaluating the effectiveness and safety of mogamulizumab in CTCL, compared mogamulizumab to vorinostat, which is not currently licensed in Europe and is not standard care in the NHS [2]. According to the CS, "vorinostat can be considered a reasonable proxy for current standard of care in the NHS, based on a naïve comparison of results from the vorinostat arm of the MAVORIC study and the physician's choice arm (methotrexate or bexarotene i.e. UK [United Kingdom] standard treatments) of the ALCANZA study as well as comparison to Phase II bexarotene data (...) [3]. It is also supported by clinical expert opinion, and the EMA [European Medicines Agency] accepted this comparison when granting marketing authorisation for mogamulizumab. Thus, the results of the MAVORIC study should be considered to translate to English clinical practice. Furthermore, vorinostat is the only drug with data in the SS population".

However, even after the response to the ERG's request for clarification, the ERG was concerned by the use of vorinostat as a comparator in MAVORIC [2]. Vorinostat 
was not standard care in the UK and was not mentioned in the proposed treatment pathway. Furthermore, based on the limited data available, the comparability of vorinostat (MAVORIC) and physician's choice (ALCANZA) could not be established $[2,3]$.

\section{Independent Evidence Review Group (ERG) Review}

The ERG reviewed the clinical effectiveness and cost-effectiveness evidence of mogamulizumab for this indication. As part of the STA process, the ERG and NICE had the opportunity to ask for clarification on specific issues in the CS, in response to which the company provided additional information [1]. The ERG also produced an ERG base case to assess the impact of alternative assumptions and parameter values on the model results, by modifying the health economic model submitted by the company. Sections 3.1-3.6 summarise the evidence presented in the CS, as well as the review of the ERG.

\subsection{Clinical Effectiveness Evidence Submitted by the Company}

A good range of databases and resources, including conference proceedings, were searched and the searches were transparent and reproducible. One set of searches was conducted to identify both efficacy and safety evidence. The searches included RCTs and observational study design filters in order to identify both efficacy and safety evidence. Searches were conducted in July 2019. Based on these literature searches, the main source of effectiveness evidence was the MAVORIC RCT [2].

PFS assessed by blinded independent review (BIR) results favoured mogamulizumab over vorinostat (hazard ratio [HR] 0.64, 95\% confidence interval [CI] 0.49-0.84). A number of measures of response rates were reported, which generally favoured mogamulizumab over vorinostat. The results for OS (BIR assessed), an exploratory outcome of the MAVORIC trial, also favoured mogamulizumab (risk difference $19.4 \%, 95 \%$ CI 9.0-29.4) [2]. The results for this outcome varied depending on the approach used to type of adjustment for switching and the censoring of participants receiving allogenic stem cell transplant (aSCT), i.e. the analysis without crossover adjustment, but censoring for aSCT favoured vorinostat, although it was not statistically significant. These methods are discussed in Sect. 3.3.

The median TTNT for mogamulizumab was statistically significantly longer than for vorinostat at 11.0 months $(95 \%$ CI 8.8-12.6) compared to 3.2 months (95\% CI 3.1-4.3). The analyses to evaluate the changes in quality of life were made using three instruments: Skindex-29, FACT-G (Functional
Assessment of Cancer Therapy-General) and EQ-5D-3L (European Quality of Life-5 Dimensions 3 levels). Results favoured mogamulizumab over vorinostat, although followup was only up to 11 cycles, i.e. less than 12 months.

The CS noted the incidence of treatment-emergent adverse events (AEs) to be comparable between the two treatment groups. The listed AEs were found to be consistently reported in the clinical study report (CSR), with the most commonly reported AEs in the mogamulizumab group being infusion-related reactions, while in the vorinostat group, this was diarrhoea and fatigue.

In response to the $\mathrm{ACD}$, the company provided new evidence and analyses, most notably an unanchored matched adjusted indirect comparison (MAIC) of mogamulizumab with UK standard care by analysing Hospital Episode Statistics (HES) data. The HES data provided real-world evidence from NHS clinical practice in all hospitals in England over a 10-year period (2009-2019), and comprises all current UK NHS patients with this very rare disease.

\subsection{Critique of Clinical Effectiveness Evidence and Interpretation}

MAVORIC was designed as an RCT, which should be considered to be an appropriate design to estimate the effectiveness of mogamulizumab versus a comparator [2]. However, as discussed in Sect. 2, there were concerns regarding the appropriateness of the comparator, vorinostat (used as a proxy for current standard of care in the NHS), with respect to the scope and UK clinical practice.

As discussed in Sect. 2, the CS used a narrower definition than that used in the NICE scope. Only "approximately $80 \%$ of patients" represented the population defined in the decision problem. Furthermore, there was some doubt as to the generalisability of the MAVORIC trial to this population [2]. Specifically, a criterion for the company decision problem population was those "who are clinically ineligible for or refractory to treatment with BV". However, even after a request for clarification, the number of participants considered to be "ineligible for BV" as well as how this status was determined remained unclear.

When estimating the effectiveness versus vorinostat, crossover (switching) from vorinostat to mogamulizumab was permitted, i.e. outcomes measured after progression such as OS could be biased. There was some additional uncertainty regarding progression assessment given that the trial was also open-label. Results were not statistically significant for MF patients and those with disease stage IIB/II. There was risk of bias for all outcomes measured after progression that resulted from the specific study design and flow of participants, where $73 \%$ of vorinostat patients switched to mogamulizumab. 
The ERG noted the warning issued by the Food and Drug Administration (FDA) for the potential complications of aSCT after mogamulizumab that may persist beyond a wash-out period of 50 days $[4,5]$. Therefore, especially in patients where allogeneic transplant is a suitable treatment option, treatment with mogamulizumab before the transplant should be carefully balanced against the potential risks.

The HES data, provided after release of the ACD, avoided two serious problems with MAVORIC: (1) vorinostat being an inappropriate comparator as it is not available in the UK, and (2) patients in the comparator arm were allowed to cross over to the mogamulizumab arm. However, there are a number of issues associated with these data. For example, the company did not provide a description of the methods used for the matching, except the process of selection of variables for which adjustment is required [6]. Based on the provided information, it appeared that data were available for only three of the variables (disease type, number of prior systemic therapies, type of prior systemic therapies) that were considered in the analysis of the HES data and only one variable (disease type) was chosen for matching. Most problematically, as stated in NICE Decision Support Unit (DSU) technical support document (TSD) 18, "an unanchored MAIC [matching adjusted indirect comparison] or STC [simulated treatment comparison] effectively assumes that absolute outcomes can be predicted from the covariates; that is, it assumes that all effect modifiers and prognostic factors are accounted for. This assumption is very strong, and largely considered impossible to meet. Failure of this assumption leads to an unknown amount of bias in the unanchored estimate" [6]. Therefore, the ERG concluded that the results of the company's HES MAIC analysis needed to be regarded with a considerable degree of caution.

\subsection{Cost-Effectiveness Evidence Submitted by the Company}

One overarching search strategy was used to identify costeffectiveness, HRQoL, and healthcare resource use evidence in several databases. In addition, targeted searches were performed in Embase and Medline to identify costeffectiveness, HRQoL, and healthcare resource use studies, and in PubMed to identify health state utilities for states describing the burden of caring for a partner with CTCL. No previous cost-effectiveness analysis of mogamulizumab was identified. Some cost and utility data were derived from the technology appraisal (TA) 577 submission (BV for treating CD30-positive CTCL) [7].

The company developed a partitioned survival model in Microsoft Excel. Two changes were made to the traditional partitioned survival approach: inclusion of the potential for patients to receive aSCT, and the use of disease control or TTNT instead of disease progression determining the health states. The inclusion of aSCT led to the modelling of three separate patient pathways: (1) patients who do not undergo aSCT, (2) patients who undergo aSCT after the current treatment, and (3) patients who undergo aSCT after subsequent treatments. These three pathways were added up to obtain the model population.

Mogamulizumab has received EMA marketing authorisation for the treatment of adult patients with MF or SS who have received at least one prior systemic therapy [8]. The patient population considered in the economic model was restricted to patients with advanced disease (stage $\geq$ IIB MF and all SS patients) only, which is a subgroup of the final scope issued by NICE and the MAVORIC trial [2,9].

As per its marketing authorisation, mogamulizumab was modelled with a posology of $1 \mathrm{mg} / \mathrm{kg}$ as an intravenous infusion administered weekly on days $1,8,15$, and 22 of the first 28 -day cycle, followed by infusions every 2 weeks on days 1 and 15 of every subsequent 28 -day cycle. Based on clinical inputs and benefits from the MAVORIC trial, a 24-month stopping rule was implemented.

UK patients with advanced MF and SS are currently treated with ECM, which comprises a number of treatments, including methotrexate, bexarotene, interferon alfa-2a, gemcitabine, a combination of cyclophosphamide, doxorubicin, vincristine, and prednisolone (CHOP), amongst others. As stated above, evidence for vorinostat was used to inform the comparator in the economic model. In response to the ACD, the company performed an MAIC of mogamulizumab with HES data [10].

The analysis took an NHS and Personal Social Services (PSS) perspective. Discount rates of 3.5\% were applied to both costs and benefits. The model cycle length was 1 week, with a time horizon of 30 years.

The main sources of evidence on treatment effectiveness used for intervention and comparators were as follows: the MAVORIC trial (patients with advanced disease); data used in TA577 from the London supra-regional centre to inform estimates of disease-free survival (DFS) and OS for patients undergoing aSCT; and expert opinion to inform proportions of patients undergoing aSCT after current treatment (mogamulizumab or ECM).

The MAVORIC study was not powered to detect OS differences between treatment arms (only 23\% of patients had an OS event) [2]. The crossover design of MAVORIC allowed patients randomised to the vorinostat arm to switch to mogamulizumab treatment if they had at least two cycles of treatment and showed confirmed disease progression or had intolerable toxicity (grade $\geq 3$ AEs, excluding inadequately treated nausea, vomiting, diarrhoea, and alopecia), despite dose reduction and appropriate management of side effects. The company stated that, given that $72.6 \%$ of patients switched from the vorinostat arm to the mogamulizumab arm, unadjusted OS data were heavily confounded 
by the crossover design. For all OS analyses, patients who received aSCT were excluded.

The company explored different crossover adjustment methods described in the NICE DSU TSD 16: the inverse probability of censoring weights (IPCW), the rank-preserving structural failure time model (RPSFTM) method, and the two-stage estimation (TSE) method [11]. The company discarded the RPSFTM method based on it producing counterintuitive OS estimates, essentially showing an OS gain for patients treated with vorinostat. Furthermore, assumptions of a common and time-invariant treatment effect underlying the RPSFTM method were likely not fulfilled. The company preferred the IPCW method over the TSE method, stating that the TSE method did not account for any spill over effects of mogamulizumab on the next treatment.

Using the IPCW method for crossover adjustment, the exponential and the generalised gamma survival models had the best statistical fit for the mogamulizumab and vorinostat arms, respectively, based on the Akaike information criterion (AIC) and Bayesian information criterion (BIC). The company chose the lognormal and the exponential models for mogamulizumab and vorinostat arms, respectively, based on fit to external data and expert opinion. Using the TSE method for crossover adjustment, the exponential model had the best statistical fit for both mogamulizumab and vorinostat arms, but the company explored the lognormal and exponential models, respectively, in their scenario analyses.

The next treatment free survival (NTFS) data were nearly complete. Parametric survival models were fitted independently, because it was uncertain whether the proportional hazard assumption held. The lognormal had the best statistical fit for the mogamulizumab arm, and the generalised gamma for the vorinostat arm. The generalised gamma was chosen for both arms in the company's base case.

The proportions of patients receiving aSCT after current treatment were based on estimates from a clinician survey, as patients in MAVORIC were not allowed to receive aSCT after current treatment. The time to receive aSCT after current treatment (18 weeks after initiation of treatment) was based on NICE TA577 [7]. A wash-out period of 50 days after stopping mogamulizumab treatment was assumed to mitigate the additional risk to patients undergoing aSCT who have had mogamulizumab. The proportions of patients receiving aSCT after subsequent treatment and the timing of aSCT were based on MAVORIC.

DFS and OS estimates for patients who received aSCT were obtained from the London supra-regional centre as reported in TA577. The company chose the best-fitting Gompertz model for DFS and the second-best-fitting lognormal model for OS to align with TA577. The mean dose intensity reported during the randomised treatment period of MAVORIC was $97.5 \%$ for mogamulizumab, and the company assumed the same dose intensity for ECM [2]. Given the mature data for the time on treatment (ToT), KaplanMeier data were used directly to capture mogamulizumab and vorinostat ToT. ToT for vorinostat was used to inform ECM ToT, except for those components of ECM that are given for a shorter duration.

The source of evidence for treatment AEs used for intervention and comparator was the safety population of the MAVORIC trial [2]. The company assumed the same AE rate for ECM as for vorinostat. Only grade 3 and 4 AEs were assumed to have an important impact on the costs and HRQoL and these were included in the model in terms of their impact on costs.

The utility values for the economic model were based on the EQ-5D-3L data from MAVORIC [2]. The UK tariff was applied to the MAVORIC EQ-5D-3L questionnaire data to generate patient-specific EQ-5D-3L utility data. The MAVORIC data were analysed using longitudinal mixed effects models; post-baseline EQ-5D utility scores were regressed on fixed effects of (1) baseline EQ-5D utility score, (2) randomised treatment, (3) current treatment, and (4) progression status (yes vs no), as well as all possible interaction terms. No differential utility value was used for the subsequent treatment health state between mogamulizumab and comparator treatment. Data from TA577 were used for utility values associated with subsequent aSCT and for end-stage care [12]. A vignette study was undertaken to evaluate caregiver utilities, in which vignettes were informed by a targeted review of qualitative studies with individuals with CTCL and/or their caregivers and interviews with CTCL specialists. Vignettes were scored by subjects from the general population and valued using the van Hout mapping algorithm [13]. A utility gain for caregivers was included for the additional time that modelled patients in the mogamulizumab arm were able to reside in the "disease control" state (compared to modelled patients in the ECM arm).

The cost categories included in the model were treatment acquisition costs, treatment administration costs, subsequent treatment costs (including aSCT, subsequent treatment costs after aSCT and subsequent treatments), and costs of managing grade 3/4 AEs. Unit costs for nondrug resources were obtained from the National Schedule of Reference Costs 2017-2018 [14] and the Personal Social Services Research Unit (PSSRU) report [15], and unit costs of drugs were obtained from the British National Formulary (BNF 2019) [16] and the Drugs and pharmaceutical electronic market information tool (eMIT 2019) [17] for generic products. Mogamulizumab was offered with a confidential discount. Resource use related to secondary care was based on the HES database, while resource use related to community care and treatments was based on published literature, including information identified by a systematic literature review, and data from previous NICE TAs and expert opinion. Subsequent 
treatments (modelled as a health state) after mogamulizumab and ECM were assumed to be equal in both arms and of equal duration, and these were derived from a clinician survey and interviews. Where necessary, costs were inflated to the 2017/18 level.

In the company's original base-case analysis, total life years (LYs) and quality-adjusted life years (QALYs) gained were larger for mogamulizumab than for ECM. Incremental QALYs (2.83) were mainly driven by QALY gains in the subsequent treatment health state, where patients in both arms spent most time. Total costs were also higher for mogamulizumab than for ECM. Incremental costs mainly resulted from higher drug costs and monitoring costs after subsequent treatment. The deterministic incremental costeffectiveness ratio (ICER) amounted to $£ 33,819$ per QALY gained. The company subsequently made changes to the model: correcting errors, incorporating some ERG preferences, but not all, and offering an increased discount. The company's revised and final ICERs were $£ 31,030$ and $£ 32,634$ per QALY gained (using the HES and MAVORIC comparison, respectively).

\subsection{Critique of Cost-Effectiveness Evidence and Interpretation}

While the ERG identified a few issues regarding the overall quality of the searches conducted, e.g. searching MEDLINE and Embase simultaneously without including both $\mathrm{MeSH}$ and EMTREE subject heading indexing terms, overall the searches were adequate, and given the range of resources searched, it was unlikely that any relevant studies were missed.

The company identified no economic evaluations addressing the decision problem it aimed to target and therefore developed a de novo economic evaluation. The company's economic evaluation met most of the NICE reference case criteria, except for the inclusion of caregivers' utilities, which were based on a vignette study.

The ERG considered that the lack of direct comparator data for the ECM arm remained a major concern in the appraisal of mogamulizumab. The ERG considered that the MAVORIC trial data, whilst coming from an RCT, was limited by crossover and uncertainty as to the most appropriate method for adjustment. On the other hand, the company's HES MAIC analysis also needed to be regarded with a considerable degree of caution and considered that it was a matter of judgement as to which of the two data sources was preferable. When using the HES analysis, the ERG preferred to use distributions for extrapolating OS and NTFS that had the better statistical fit.

The partitioned survival analysis using different pathways to reflect the possibility of patients receiving aSCT was deemed problematic, as in MAVORIC, patients were not eligible for aSCT after current treatment. To include aSCT after current treatment in the model, the company therefore added a new cohort of patients. The problem with this approach was that this cohort had zero risk of mortality and remained in the disease control state until aSCT, thereby inflating the survival benefit of patients in MAVORIC. The ERG preferred to disable the aSCT after current treatment pathway.

OS was based on MAVORIC, but it was only an exploratory, not a primary, endpoint. As such, MAVORIC was not powered to estimate OS, and maturity was not achieved [2]. All OS extrapolations were therefore highly uncertain. The choice of parametric survival model for extrapolation of OS had a large impact on model outcomes and was associated with substantial uncertainty. In addition, the comparator OS estimates derived from MAVORIC were confounded by crossover, which required adjustment in statistical analyses. Different adjustment methods had vastly different results. Using the RPSFTM method, the difference between vorinostat and mogamulizumab OS (which favoured vorinostat) became even larger, as RPSFTM assumed that treatment multiplies survival time. These results did not appear plausible to clinical experts, and the RPSFTM was ruled out. It is worth noting that both TSE and IPCW methods rely on assumptions: both require there to be no unmeasured confounders and, in addition, the TSE requires the existence of as secondary baseline, that is, the time/cut-off point after which switching was allowed. The ERG noted that progression status was one of the main criteria for switching, and the majority of patients appeared to have switched because of progression, which supported the existence of a secondary baseline. Furthermore, for the TSE method, the "no unmeasured confounders" assumption is important at the time of the secondary baseline, which may be more easily satisfied than at other time points where other variables may not have been measured (required for the IPCW method). For the IPCW method, it is also important that the proportion of patients who did not switch out of those eligible for switching is not too low, or else large weights may be obtained for a small number of patients. The company did not provide the proportion of patients who did not switch out of those eligible for switching. The proportion of patients switching in the advanced population was $71.5 \%$ (133 patients in that subgroup). The company took account of Eastern Cooperative Oncology Group (ECOG) status, progression status, age, and time in their weighting. It appeared that some extreme weights $(>10)$ were obtained when using the IPCW method, although the company did not present all information on their analysis and results. According to TSD 16 and other literature, weights larger than 10 could mean that the IPCW method produces biased results [11, 18, 19]. In addition, the ERG's clinical 
expert considered that the TSE method produced more plausible OS estimates than the IPCW method considering the observed MAVORIC data. However, it was unclear whether the company used re-censoring in its application of the TSE method. The ERG also considered that there was no evidence supporting any spill-over effects of mogamulizumab treatment to subsequent treatment. The ERG therefore used the TSE method in its base case. The ERG acknowledged that there was uncertainty about the most appropriate method and used the IPCW method in an exploratory analysis.

The inclusion of caregiver utilities, whilst not unprecedented, lacked guidance on whether their inclusion was appropriate and, if so, how this should be done. The ERG was concerned that the utility values were obtained using a vignette study, which did not meet the NICE reference case. However, the ERG acknowledged that the company's approach of incorporating caregiver utilities in the model avoided commonly encountered limitations, by only considering the incremental advantage on utility values for a certain time horizon. The ERG did not consider caregiver utilities in the base case, but considered that their inclusion may be a relevant scenario.

The implementation of a 24-month stopping rule was not in line with the MAVORIC trial or the licence, and the ERG therefore preferred not to use it.

\subsection{Additional Work Undertaken by the ERG}

The ERG made multiple changes to the model, including the correction of minor modelling errors. In addition, the stopping rule, aSCT after current treatment, and caregiver utilities, were disabled. The ERG also chose alternative distributions for the modelling of OS, NTFS, and DFS after aSCT. Further exploratory analyses were also performed, most notably on the choice of crossover adjustment method including model averaging (70\% TSE, 30\% IPCW, proportions based on ERG assumption), using the intent-to-treat population instead of the advanced population, and including caregiver utilities. Based on the company's HES data scenario, the ERG amended distributions for OS and NTFS to match its preferences. It is important to note that both the company's and ERG's ICERs suffered from large uncertainty and should be interpreted with caution. The ERG's final base-case ICERs were $£ 38,274$ and $£ 80,555$ per QALY gained (using the HES and MAVORIC comparison, respectively). Some of the ERG's scenarios lowered the ICER significantly (though ICERs still remained above $£ 30,000$ per QALY gained), most notably the inclusion of caregiver utilities and the choice of the IPCW method in the MAVORIC analysis.

\subsection{Conclusions of the ERG Report and Appraisal Consultation phase}

The CS was overall of high quality and transparently described. The company's model was functional and allowed for exploration of multiple relevant scenarios. The submission was complete in terms of the studies included and data used and was mostly in line with the NICE reference case. Overall, the company's ICER was regarded as very uncertain and likely biased, a product of issues with the evidence, uncertainty about methods, and model assumptions. The main driver of cost-effectiveness was OS. This was uncertain because of data immaturity, a large number of patients switching treatment in the main trial, and the lack of appropriate evidence for the comparator when using evidence from MAVORIC, and potential biases in the MAIC using the HES data.

\section{Key Methodological Issues}

Vorinostat, which is not standard care in the UK, was used as a comparator, and the company aimed to establish comparability of vorinostat to physician's choice (as a proxy for ECM) using two separate trials and data from the HES database. Uncertainty remained regarding the appropriateness of this approach, e.g. in line with the guidance of DSU TSD 18.

When adjusting for crossover, full documentation should be provided in line with guidance [19]. More guidance on the choice of most suitable crossover adjustment method may be helpful.

As stated in the NICE DSU Method report, it remains unclear when and how carer health effects should be included in economic evaluations [20]. Urgent guidance is required. An approach of only considering the incremental advantage on utility values for a certain time horizon (for example, for a specific health state) may be a way forward.

\section{National Institute for Health and Care Excellence Guidance}

On March 4, 2021, NICE determined that mogamulizumab was not recommended for treating MF or SS in adults who have had at least one previous systemic treatment. The clinical trial evidence was considered very uncertain because mogamulizumab was compared with vorinostat, a treatment that is not used or licensed in the UK. Also, many people switched treatments and there were a lot of differences among the trial population. Indirectly comparing the mogamulizumab trial evidence with evidence from patients having treatment in the NHS in England was also regarded as uncertain. 


\subsection{Consideration of Clinical Effectiveness}

The AC was concerned about using the provided clinical effectiveness data because vorinostat was not licensed for use in the UK and did not represent NHS standard care [21]. Additional uncertainty was caused by a large number of patients switching treatments. Therefore, the AC considered that the evidence for mogamulizumab was limited and concluded that its relative treatment effect compared with NHS standard care was uncertain.

Regarding the HES analysis, the AC recognised that this addressed some of the issues with the original submission and commended the company on its efforts [21]. But the limitations of the data, the lack of information on prognostic factors, and the difficulty in assessing its reliability meant that the HES analysis results were uncertain. The committee was unable to draw a conclusion about the relative treatment efficacy of mogamulizumab compared with standard care based on this analysis.

Considering whether end of life criteria were met, the AC was not convinced there was robust evidence that the short life expectancy criterion had been met. It concluded that mogamulizumab could not be considered a life-extending treatment at the end of life [21].

\subsection{Consideration of Cost-Effectiveness}

The AC considered that the model structure was acceptable, but that there was uncertainty in the data sources [21]. It recognised that some people may have aSCT in clinical practice. In the model, it preferred removing aSCT after current treatment, to avoid double-counting survival benefit in MAVORIC and to reduce potential bias. The AC was not convinced that mogamulizumab provided a prolonged benefit after disease progression and could be considered disease-modifying. It recognised that the choice of crossover adjustment had a large effect on the cost-effectiveness results. It concluded that the results from the TSE and IPCW methods represented the upper and lower range of plausible OS in the standard care arm. The AC considered that all extrapolations were uncertain, but considered the ERG's preferred and best-fitting exponential model for OS in both treatment arms as appropriate in the MAVORIC analysis. In the HES analysis, the AC agreed with the company's approach of extrapolating OS. It considered the application of a 24-month stopping rule inappropriate.

The AC considered that the company's approach for including caregiver utilities was not robust because the utility gain in the base case for carers was implausibly large compared with the expected utility gain for people with the condition. It recognised that there was a lack of detailed methodology on how to model carer utility. But it noted that the company used vignettes in the general population, which was not in line with NICE's guide to the methods of TA. It preferred to remove caregiver utilities from the base-case analysis, but recognised the burden placed on some carers.

\section{Conclusions}

Mogamulizumab compared to vorinostat showed favourable results in patients with MF and SS. However, the ERG was concerned by the use of vorinostat as a comparator in MAVORIC as it was not standard care in the UK. Based on the limited data available, the comparability of vorinostat (MAVORIC) and physician's choice (ALCANZA) cannot be established. In response to the ACD, the company provided additional analyses, based on the HES database. In principle, this addressed some of the ERG's concerns. However, the ERG identified a high risk of bias with these analyses, which should therefore be regarded with a considerable degree of caution. Therefore, the ERG had a preference for the original analysis based on MAVORIC data. These concerns were shared by the AC.

The AC did not recommend mogamulizumab for treating MF or SS in adults who have had at least one previous systemic treatment recommended. The AC noted the high level of uncertainty associated with the MAVORIC analysis, specifically:

- The relative treatment effect of mogamulizumab compared with NHS standard care was uncertain because MAVORIC did not include the appropriate comparator.

- The company's preferred subgroup was limited because it included a mixed population in a single post hoc analysis.

- There was a high level of crossover, adjustments were potentially biased, and the methods produced a wide range of estimates of treatment effect.

- The OS data were immature. Also, OS was not a primary endpoint in MAVORIC, so the trial was not powered to estimate differences.

Although the HES analysis addressed some of the issues associated with MAVORIC (for example, comparator and crossover adjustment), the committee noted it was also associated with uncertainty. It recalled that the ERG could not assess the reliability of the HES analysis, and because of data limitations, only one prognostic factor had been matched. Therefore, it agreed that an acceptable ICER would be no higher than the middle of the range normally considered a cost-effective use of NHS resources $(£ 20,000-£ 30,000$ per QALY gained). The committee concluded that, based on its preferred assumptions, all ICERs were much higher than the middle of the range normally considered cost-effective. 
Therefore, mogamulizumab could not be recommended for routine use in the NHS.

Acknowledgements This summary of the ERG report was compiled after NICE issued the FAD. All authors have commented on the submitted manuscript and have given their approval for the final version to be published. The views and opinions expressed therein are those of the authors and do not necessarily reflect those of NICE or the Department of Health. Any errors are the responsibility of the authors.

Project support was provided by Regina Leadley, Vanesa Huertas Carrera, and Gill Worthy, all from KSR.

Author contributions All authors have commented on the submitted manuscript and have given their approval for the final version to be published. RW, AC, CA, and JK critiqued the clinical effectiveness data reported by the company. S. Duffy critiqued the literature searches undertaken by the company. S. Ryder critiqued the statistical analyses performed by the company. IS provided clinical expert opinion on all aspects of this appraisal. SG, WW, MH, BW, NA, and MJ critiqued the mathematical model provided and the cost-effectiveness analyses submitted by the company. SG acts as overall guarantor for the article. This summary has not been externally peer reviewed by PharmacoEconomics.

\section{Declarations}

Funding This project was funded by the National Institute for Health Research (NIHR) Health Technology Assessment Programme. Please visit the HTA programme website for further project information (https://www.nihr.ac.uk/funding-and-support/funding-for-researchstudies/funding-programmes/health-technologyassessment).

Conflict of interest SG, WW, RW, AC, MH, BW, CA, SR, NA, SD, IS, $\mathrm{JK}$, and $\mathrm{MJ}$ have no conflicts of interest to declare.

Ethics approval Not applicable.

Consent to participate Not applicable.

Consent for publication Not applicable.

Availability of data and material Data sharing not applicable to this article as no datasets were generated or analysed during the current study.

Code availability Not applicable.

Open Access This article is licensed under a Creative Commons Attribution-NonCommercial 4.0 International License, which permits any non-commercial use, sharing, adaptation, distribution and reproduction in any medium or format, as long as you give appropriate credit to the original author(s) and the source, provide a link to the Creative Commons licence, and indicate if changes were made. The images or other third party material in this article are included in the article's Creative Commons licence, unless indicated otherwise in a credit line to the material. If material is not included in the article's Creative Commons licence and your intended use is not permitted by statutory regulation or exceeds the permitted use, you will need to obtain permission directly from the copyright holder. To view a copy of this licence, visit http://creativecommons.org/licenses/by-nc/4.0/.

\section{References}

1. National Institute for Health and Care Excellence. Mogamulizumab for previously treated mycosis fungoides and Sézary syndrome [ID1405]. London: National Institute for Health and Care Excellence. 2021. https://www.nice.org.uk/guidance/indevelopm ent/gid-ta10305/documents. Accessed 7 Jun 2021.

2. Kim YH, Bagot M, Pinter-Brown L, Rook AH, Porcu P, Horwitz SM, et al. Mogamulizumab versus vorinostat in previously treated cutaneous T-cell lymphoma (MAVORIC): an international, open-label, randomised, controlled phase 3 trial. Lancet Oncol. 2018;19(9):1192-204.

3. Prince HM, Kim YH, Horwitz SM, Dummer R, Scarisbrick J, Quaglino P, et al. Brentuximab vedotin or physician's choice in CD30-positive cutaneous T-cell lymphoma (ALCANZA): an international, open-label, randomised, phase 3, multicentre trial. Lancet. 2017;390(10094):555-66.

4. US Food and Drug Administration. Poteligeo (mogamulizumabkpkc). Labeling [Internet]: US Food and Drug Administration, 2018. https://www.accessdata.fda.gov/drugsatfda_docs/nda/2018/ 761051Orig1s000Lbl_.pdf. Accessed 30 Jan 2020

5. Fuji S, Inoue Y, Utsunomiya A, Moriuchi Y, Uchimaru K, Choi I, et al. Pretransplantation anti-CCR4 antibody mogamulizumab against adult T-cell leukemia/lymphoma is associated with significantly increased risks of severe and corticosteroid-refractory graft-versus-host disease, nonrelapse mortality, and overall mortality. J Clin Oncol. 2016;34(28):3426-33.

6. Phillipo D, Ades AE, Dias S, Palmer S, Abrams KR, Welton NJ. NICE DSU technical support document 18: methods for population-adjusted indirect comparisons in submissions to NICE [Internet]. Sheffield: Decision Support Unit, School of Health and Related Research, University of Sheffield (ScHARR), 2016. Accessed 21 Jan 2020.

7. National Institute for Health and Care Excellence. Brentuximab vedotin for treating CD30-positive cutaneous T-cell lymphoma. NICE technology appraisal guidance 577. Final appraisal document. London: National Institute for Health and Care Excellence. 2019. https://www.nice.org.uk/guidance/ta577/documents/finalappraisal-determination-document. Accessed 21 Jan 2020.

8. European Medicines Agency. Poteligeo $4 \mathrm{mg} / \mathrm{mL}$ concentrate for solution for infusion: EPAR - Product Information. Annex I. Summary of product characteristics. Amsterdam: European Medicines Agency.s 2019. https://www.ema.europa.eu/en/documents/ product-information/poteligeo-epar-product-information_en.pdf. Accessed 21 Jan 2020.

9. National Institute for Health and Care Excellence. Mogamulizumab for treated mycosis fungoides or Sézary syndrome cutaneousT-cell lymphoma. Final scope. London: National Institute for Health and Care Excellence. 2019. https://www.nice.org.uk/ guidance/gid-ta10305/documents/final-scope. Accessed 21 Jan 2020

10. NHS Digital. Hospital Episode Statistics (HES). NHS Digital. 2019. https://digital.nhs.uk/data-and-information/data-tools-andservices/data-services/hospital-episode-statistics. Accessed 21 Jan 2020.

11. Latimer N, Abrams K. NICE DSU technical support document 16: adjusting survival time estimates in the presence of treatment switching. Sheffield: NICE Decision Support Unit. 2014. http:// www.nicedsu.org.uk. Accessed 21 Jan 2020.

12. National Institute for Health and Care Excellence. Brentuximab vedotin for treating CD30-positive cutaneous T-cell lymphoma. NICE technology appraisal guidance 577. London: National Institute for Health and Care Excellence. 2019. https://www.nice.org. uk/guidance/TA577. Accessed 7 Jun 2021. 
13. van Hout B, Janssen MF, Feng YS, Kohlmann T, Busschbach $\mathrm{J}$, Golicki D, et al. Interim scoring for the EQ-5D-5L: mapping the EQ-5D-5L to EQ-5D-3L value sets. Value Health. 2012;15(5):708-15.

14. NHS Improvement. Reference costs 2017/18. London: NHS Improvement. 2018. https://improvement.nhs.uk/resources/refer ence-costs/. Accessed 21 Jan 2020.

15. Personal Social Services Research Unit. Unit costs of health and social care 2018. Canterbury: University of Kent, 2018. https:// www.pssru.ac.uk/project-pages/unit-costs/unit-costs-2018/. Accessed 21 Jan 2020.

16. Joint Formulary Committee. British National Formulary. London: BMJ Group and Pharmaceutical Press. 2019. https://bnf.nice.org. uk/. Accessed 21 Jan 2020.

17. Department of Health and Social Care. Drugs and pharmaceutical electronic market information tool (eMIT). London: Department of Health and Social Care. 2019. https://www.gov.uk/government/ publications/drugs-and-pharmaceutical-electronic-market-infor mation-emit. Accessed 21 Jan 2020.

18. Latimer NR, Abrams KR, Lambert PC, Crowther MJ, Wailoo AJ, Morden JP, et al. Adjusting for treatment switching in randomised controlled trials - a simulation study and a simplified two-stage method. Stat Methods Med Res. 2017;26(2):724-51.

19. Sullivan TR, Latimer NR, Gray J, Sorich MJ, Salter AB, Karnon J. Adjusting for treatment switching in oncology trials: a systematic review and recommendations for reporting. Value Health. 2020;23(3):388-96.

20. Pennington B, Wong R. Modelling carer health-related quality of life in NICE Technology Appraisals and Highly Specialised Technologies. Sheffield: Decision Support Unit, School of Health and Related Research, University of Sheffield (ScHARR). 2019. http://nicedsu.org.uk/modelling-carer-health-related-quality-oflife/. Accessed 7 Jun 2021.

21. National Institute for Health and Care Excellence. Mogamulizumab for previously treated mycosis fungoides and Sézary syndrome. Final appraisal document. London: National Institute for Health and Care Excellence. 2021. https://www.nice.org.uk/guida nce/gid-ta10305/documents/final-appraisal-determination-docum ent. Accessed 28 Jun 2021.

\section{Authors and Affiliations}

\section{Sabine E. Grimm ${ }^{1}\left(\right.$ ) Willem Witlox ${ }^{1} \cdot$ Robert Wolff $^{2} \cdot$ Annette Chalker $^{2} \cdot$ Mickael Hiligsmann $^{3} \cdot$ Ben Wijnen $^{1}$. Charlotte Ahmadu ${ }^{2}$. Steve Ryder ${ }^{2} \cdot$ Nigel Armstrong $^{2} \cdot$ Steven Duffy $^{2} \cdot$ Isabel Syndikus $^{4}$ - Jos Kleijnen ${ }^{2,3}$. Manuela A. Joore ${ }^{1}$}

\section{Sabine E. Grimm} sabine.grimm@mumc.nl

1 Department of Clinical Epidemiology and Medical Technology Assessment, Maastricht University Medical Centre+ (MUMC+), P. Debyelaan 25, PO Box 5800, 6202 AZ Maastricht, The Netherlands

2 Kleijnen Systematic Reviews Ltd, York, UK
3 Department of Health Services Research, Care and Public Health Research Institute (CAPHRI), Maastricht University, Maastricht, The Netherlands

4 The Clatterbridge Cancer Centre National Health Service (NHS) Foundation Trust, Wirral, UK 